\title{
The first law of black-hole thermodynamics for black holes in string theory
}

\author{
Emre Sermutlu† \\ Department of Physics and Astronomy, University of Pennsylvania, Philadelphia, PA 19104, \\ USA
}

Received 21 November 1997, in final form 10 March 1998

\begin{abstract}
We investigate thermodynamical properties of four- and five-dimensional black-hole solutions of toroidally compactified string theory. We find an explicit expression for the first law of black-hole thermodynamics. We calculate the temperature $\mathrm{T}$, angular velocity $\bullet$ and the electromagnetic potentials $8_{\mathrm{i}}$ on the horizon using two different methods.

PACS numbers: 0470D, 1125
\end{abstract}

\section{Introduction}

The connection between black-hole mechanics and thermodynamics is one of the most interesting developments in the past 30 years. The first law of black-hole mechanics, as proved by Bardeen et al [1], gives the variation of mass in terms of the variations in area and angular momentum. This relationship opened the way for the area of the black hole to be interpreted as its entropy and the surface gravity as its temperature.

With the discovery of the Hawking radiation, it was understood that the close parallel between the laws of thermodynamics and black-hole mechanics was more than a coincidence and had a physical basis. Black holes radiate with a black-body spectrum at the temperature given by surface gravity.

Hawking radiation, while answering an important question, raised new ones like information loss, black-hole evaporation and the microscopic origin of black-hole entropy.

In this paper, I am interested in finding the explicit form of the first law of black-hole mechanics for two different black holes. In other words, given the metric, I want to find the coefficients $\mathrm{T}, \bullet$ and 8 in

$$
\mathrm{dM}=\mathrm{T} \mathrm{dS}+\cdot \mathrm{dJ}+8_{\mathrm{i}} \mathrm{dQ}_{\mathrm{i}}
$$

If we have a black-hole solution, we can easily calculate the surface area of the outer horizon using the metric components. Entropy is given by $S=\left(1 / 4 \mathrm{G}_{\mathrm{N}}\right) \mathrm{A}$. Then, if the algebraic equations are tractable, we can isolate $\mathrm{M}$, take derivatives, and obtain the first law.

This procedure will not work if the solution is given in terms of parameters that cannot be solved explicitly in terms of mass and charges. Then we have to use a roundabout

† E-mail address: sermutlu@cvetic.hep.upenn.edu 
On leave from: Department of Mathematics, Bilkent University, Ankara, Turkey.

E-mail address: sermutlu@fen.bilkent.edu.tr

0264-9381/98/061449+13\$19.50 C) 1998 IOP Publishing Ltd

way, using infinitesimal variations to make a change of variables, which in general involve inverting a big matrix, and if all entries are non-zero, the results may be too complicated.

We expect the variation of mass with respect to area to be the temperature of the black hole, and the variation of mass with respect to angular momentum to be the angular velocity. These quantities can be computed from the metric. This will be an independent way to calculate the coefficients in the first law, and will provide a check on the results.

In this paper, we will follow the summarized procedure for two different types of black hole, corresponding to four- and five-dimensional solutions of toroidally compactified string theory. We first write the area in terms of solution parameters, take the infinitesimal variation of the area, and replace the solution parameters by the physical ones using the Jacobian matrix. Then we calculate $\bullet$ and $\kappa$ using the metric, and compare the results with the first law.

I have also tried to obtain Smarr's formula [2], but we do not know the mass as an explicit function, so Smarr's procedure of using Euler's theorem to obtain $\mathrm{M}=2 \mathrm{TA}+2 \cdot \mathrm{J}+8 \mathrm{Q}$ is not applicable in this case.

In section 2, a four-dimensional rotating black hole parametrized by ADM mass, four charges, and angular momentum [3] will be analysed, and in section 3 we consider a fivedimensional black hole with two angular momenta and three charges [4].

\section{Four dimensions}

\subsection{The metric and physical parameters}

The metric for four-dimensional rotating charged black-hole solutions of toroidally compactified superstring theory, parametrized by the ADM mass, four charges and angular momentum, given by $\quad$ is $\quad$ [3] $s_{E}^{2}=\Delta^{1 / 2}\left[\begin{array}{c}r^{2}-2 m r+l^{2} \cos ^{2} \theta \\ \Delta\end{array} \mathrm{d} t^{2}+r^{2}-2 m r+l^{2}+\mathrm{d} \theta^{2}\right.$

$$
\begin{aligned}
& +\frac{\sin ^{2} \theta}{\Delta}\left\{\left(r+2 m \sinh ^{2} \delta_{1}\right)\left(r+2 m \sinh ^{2} \delta_{2}\right)\left(r+2 m \sinh ^{2} \delta_{3}\right)\right. \\
& \left.\times\left(r+2 m \sinh ^{2} \delta_{4}\right)+l^{2}\left(1+\cos ^{2} \theta\right) r^{2}+W+2 m l^{2} r \sin ^{2} \theta\right\} \mathrm{d} \phi^{2} \\
& -\frac{4 m l}{\Delta}\left\{\left(\cosh \delta_{1} \cosh \delta_{2} \cosh \delta_{3} \cosh \delta_{4}-\sinh \delta_{1} \sinh \delta_{2} \sinh \delta_{3} \sinh \delta_{4}\right) r\right. \\
& \left.\left.+2 m \sinh \delta_{1} \sinh \delta_{2} \sinh \delta_{3} \sinh \delta_{4}\right\} \sin ^{2} \theta \mathrm{d} t \mathrm{~d} \phi\right],
\end{aligned}
$$

where

$$
\begin{aligned}
1 \equiv\left(\mathrm{r}+2 \mathrm{msinh}^{2} \delta_{1}\right)\left(\mathrm{r}+2 \mathrm{msinh}^{2} \delta_{2}\right)\left(\mathrm{r}+2 \mathrm{msinh}^{2} \delta_{3}\right)\left(\mathrm{r}+2 \mathrm{msinh}^{2} \delta_{4}\right) \\
+\left(2 \mathrm{l}^{2} \mathrm{r}^{2}+\mathrm{W}\right) \cos ^{2} \theta \\
\mathrm{W} \equiv 2 \mathrm{ml}^{2}\left(\sinh ^{2} \delta_{1}+\sinh ^{2} \delta_{2}+\sinh ^{2} \delta_{3}+\sinh ^{2} \delta_{4}\right) \mathrm{r}
\end{aligned}
$$


$-2 \sinh ^{2} \delta_{1} \sinh ^{2} \delta_{2} \sinh ^{2} \delta_{3} \sinh ^{2} \delta_{4}-\sinh ^{2} \delta_{1} \sinh ^{2} \delta_{2} \sinh ^{2} \delta_{4}$

$-\sinh ^{2} \delta_{1} \sinh ^{2} \delta_{2} \sinh ^{2} \delta_{3}-\sinh ^{2} \delta_{2} \sinh ^{2} \delta_{3} \sinh ^{2} \delta_{4}-$

$\left.\sinh ^{2} \delta_{1} \sinh ^{2} \delta_{3} \sinh ^{2} \delta_{4}\right)+1^{4} \cos ^{2} \theta$.

The outer and inner event horizons are at

$$
\mathrm{r}_{ \pm}=\mathrm{m} \pm \overline{\mathrm{pm} 2-12}
$$

Here, $\mathrm{m}$, the non-extremality parameter, is related to the mass of the Kerr solution, 1 is related to the angular momentum of the Kerr solution and $\delta_{1,2,3,4}$ are boost parameters. Our aim is to write the variation of $\mathrm{S}$ in terms of the physical parameters ADM mass $\mathrm{M}$, two electric charges $\mathrm{Q}_{1}, \mathrm{Q}_{2}$, two magnetic charges $\mathrm{P}_{1}, \mathrm{P}_{2}$, and the angular momentum $\mathrm{J}$. The physical parameters can be expressed in terms of $\mathrm{m}, 1$ and the boosts as follows:

$M=4 m\left(\cosh ^{2} \delta_{1}+\cosh ^{2} \delta_{2}+\cosh ^{2} \delta_{3}+\cosh ^{2} \delta_{4}\right)-8 m$,

$\mathrm{Q}_{1}=4 \mathrm{~m} \cosh \delta_{1} \sinh \delta_{1}$,

$\mathrm{Q}_{2}=4 \mathrm{~m} \cosh \delta_{2} \sinh \delta_{2}$,

$\mathrm{P}_{1}=4 \mathrm{~m} \cosh \delta_{3} \sinh \delta_{3}$

$\mathrm{P}_{2}=4 \mathrm{mcosh} \delta_{4} \sinh \delta_{4}$

$\mathrm{J}=8 \operatorname{lm}\left(\cosh \delta_{1} \cosh \delta_{2} \cosh \delta_{3} \cosh \delta_{4}-\sinh \delta_{1} \sinh \delta_{2} \sinh \delta_{3} \sinh \delta_{4}\right)$

Note that we choose $G_{N}=\frac{1}{4} \pi$.

\subsection{The first law of black-hole thermodynamics}

The entropy is given by $\left(1 / 4 \mathrm{G}_{\mathrm{N}}\right) \mathrm{A}$ where $\mathrm{A}$ is the area of the outer horizon. In this case, $\mathrm{S}$ has the form [3]:

$S=16 \pi\left[\left(m^{2}+m \sqrt{m^{2}-l^{2}}\right)\left(\cosh \delta_{1} \cosh \delta_{2} \cosh \delta_{3} \cosh \delta_{4}\right)\right.$

$$
\left.+\left(m^{2}-m \sqrt{m^{2}-l^{2}}\right)\left(\sinh \delta_{1} \sinh \delta_{2} \sinh \delta_{3} \sinh \delta_{4}\right)\right] .
$$

We can write the variation of entropy in terms of the solution parameters as follows:

$$
\mathrm{d} S=\frac{\partial S}{\partial \delta_{1}} \mathrm{~d} \delta_{1}+\frac{\partial S}{\partial \delta_{2}} \mathrm{~d} \delta_{2}+\frac{\partial S}{\partial \delta_{3}} \mathrm{~d} \delta_{3}+\frac{\partial S}{\partial \delta_{4}} \mathrm{~d} \delta_{4}+\frac{\partial S}{\partial m} \mathrm{~d} m+\frac{\partial S}{\partial l} \mathrm{~d} l,
$$

but we want to write the variation in terms of the physical parameters:

$$
\mathrm{dS}=0_{1} \mathrm{dQ}_{1}+0_{2} \mathrm{dQ}_{2}+0_{3} \mathrm{dP}_{1}+0_{4} \mathrm{dP}_{4}+0_{5} \mathrm{dM}+0_{6} \mathrm{dJ}
$$

where 
$\Gamma_{1}=\left(\frac{\partial S}{\partial \delta_{1}} \frac{\partial \delta_{1}}{\partial Q_{1}}+\frac{\partial S}{\partial \delta_{2}} \frac{\partial \delta_{2}}{\partial Q_{1}}+\frac{\partial S}{\partial \delta_{3}} \frac{\partial \delta_{3}}{\partial Q_{1}}+\frac{\partial S}{\partial \delta_{4}} \frac{\partial \delta_{4}}{\partial Q_{1}}+\frac{\partial S}{\partial m} \frac{\partial m}{\partial Q_{1}}+\frac{\partial S}{\partial l} \frac{\partial l}{\partial Q_{1}}\right)$,

etc. By rearranging (8), we can now write the variation of $M$ to obtain the explicit form of the first law for this black hole:

$$
\mathrm{dM}=\mathrm{T} \mathrm{dS}+\cdot \mathrm{dJ}+8_{1} \mathrm{dQ}_{1}+8_{2} \mathrm{dQ}_{2}+8_{3} \mathrm{dP}_{1}+8_{4} \mathrm{dP}_{2},
$$

To find the $0_{\mathrm{i}}$ 's, we need the derivatives of the solution parameters $\left(\delta_{\mathrm{i}}\right.$ 's etc) with respect to the physical parameters $\left(\mathrm{Q}_{\mathrm{i}}\right.$ 's etc). We cannot invert equation (5), so we cannot find the solution parameters explicitly in terms of the physical parameters. But we can always find the infinitesimal variations by inverting the Jacobian matrix. The details of this lengthy but straightforward calculation are in appendix A.

Thus, we can find the coefficients in (10) in terms of $\mathrm{S}$ as:

$$
\begin{aligned}
& T=\frac{4 \sqrt{m^{2}-l^{2}}}{S}, \\
& \Omega=\frac{8 \pi l}{S}, \\
& \Phi_{i}=\frac{S_{i}}{S} .
\end{aligned}
$$

In [2] Smarr obtained the formula $\mathrm{dM}=\mathrm{T} \mathrm{dA}+\cdot \mathrm{dJ}+8 \mathrm{dQ}$ and from this, using the fact that $\mathrm{M}$ is homogeneous of degree $\frac{1}{2}$ in $\left(A, L, Q^{2}\right)$, he obtained

$$
\mathrm{M}=2 \mathrm{TA}+2 \cdot \mathrm{J}+8 \mathrm{Q} .
$$

However, we are not in a position to repeat this, because we cannot express $M$ in terms of area and charges explicitly, so we are unable to find the analogue of Smarr's formula (12).

\subsection{Thermodynamical quantities derived from the metric}

We now determine the thermodynamical quantities in (10) using the metric. The temperature $\mathrm{T}$ is related to the surface gravity $\kappa$ by

$$
2 \pi T=\kappa=-\left.\frac{1}{2} \frac{\mathrm{d} g_{t t}}{\mathrm{~d} r}\right|_{r=r_{+}, \theta=0}
$$

d

Using the metric

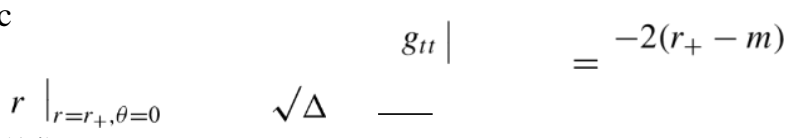

(14)

$\mathrm{d}$

where

$$
\left.\sqrt{\Delta}\right|_{r=r_{+}, \theta=0}=2 m\left(m K+\sqrt{m^{2}-l^{2}} L\right)=\frac{S}{8 \pi}
$$

Thus, 


$$
\kappa=\frac{8 \pi \sqrt{m^{2}-l^{2}}}{S},
$$

which is in agreement with the first law (10).

The angular velocity of the black hole at the outer horizon is:

$$
\left.\Omega \equiv \frac{-g_{t t}}{g_{\phi t}}\right|_{r=r_{+}, \theta=0 .}
$$

From the metric (2) we can write

(18) $r=r_{+}=m+\sqrt{ } m^{2}-l^{2}$

$$
\frac{g_{t t}}{g_{\phi t}}=\frac{r^{2}-2 m r+l^{2}-l^{2} \sin ^{2} \theta}{2 m l \sin ^{2} \theta(r L+m K-m L)} .
$$

At

$$
\begin{aligned}
& \text { the }\left.{ }^{\frac{g_{t t}}{g_{\phi t}}}\right|_{r=r_{+}, \theta=0}=\frac{-l}{2 m\left(\sqrt{m^{2}-l^{2}} L+m K\right)}=\frac{-8 \pi l}{S} \\
& \text { (19) } \Omega_{H}=\frac{8 \pi l}{S},
\end{aligned}
$$

which is also in agreement with the first law (10).

\section{Black holes in five dimensions}

\subsection{Metric and physical parameters}

The metric for five-dimensional rotating charged black holes of toroidally compactified string theory, specified by the ADM mass $\mathrm{M}$, three charges $\mathrm{Q}_{1}, \mathrm{Q}_{2}, \mathrm{Q}_{3}$ and two rotational parameters $1_{1}, 1_{2}$, is given by [4]: $\mathrm{d}_{E}^{2}=g_{t t} \mathrm{~d} t^{2}+g_{r r} \mathrm{~d} r^{2}+g_{\theta \theta} \mathrm{d} \theta^{2}+2 g_{\phi \psi} \mathrm{d} \phi \mathrm{d} \psi+2 g_{\phi t} \mathrm{~d} \phi \mathrm{d} t$

$$
+2 \mathrm{~g}_{\psi \mathrm{t}} \mathrm{d} \psi \mathrm{dt}+\mathrm{g}_{\varphi \varphi} \mathrm{d} \varphi^{2}+\mathrm{g}_{\psi \psi} \mathrm{d} \psi^{2},
$$

$g_{t t}=-\Delta^{-2 / 3} R(R-2 m)$,

$$
\begin{aligned}
g_{r r} & =\frac{\Delta^{1 / 3} r^{2}}{\left(r^{2}+l_{1}^{2}\right)\left(r^{2}+l_{2}^{2}\right)-2 m r^{2}} \\
g_{\theta \theta} & =\Delta^{1 / 3}, \\
g_{\phi \psi} & =\cos ^{2} \theta \sin ^{2} \theta \Delta^{-2 / 3}\left(L_{1} k_{3}+L_{3} k_{1}\right), \\
g_{\phi t} & =-2 m \sin ^{2} \theta \Delta^{-2 / 3}\left(l_{1} R c_{1} c_{2} c_{3}+l_{2}(2 m-R) s_{1} s_{2} s_{3}\right), \\
g_{\psi t} & =-2 m \cos ^{2} \theta \Delta^{-2 / 3}\left(l_{1}(2 m-R) s_{1} s_{2} s_{3}+l_{2} R c_{1} c_{2} c_{3}\right. \\
g_{\phi \phi} & =\sin ^{2} \theta \Delta^{-2 / 3}\left[\Delta+\sin ^{2} \theta\left(L_{1} k_{1}+L_{2} k_{2}+L_{3} k_{3}\right)\right], \\
g_{\psi \psi} & =\cos ^{2} \theta \Delta^{-2 / 3}\left[\Delta+\cos ^{2} \theta\left(L_{1} k_{1}-L_{2} k_{2}+L_{3} k_{3}\right)\right],
\end{aligned}
$$


where

$$
\begin{aligned}
& \mathrm{L}_{1}=\mathrm{l}_{12}+\mathrm{l}_{22}, \\
& \mathrm{~L}_{2}=\mathrm{l}_{12}-\mathrm{l}_{22}, \mathrm{~L}_{3} \\
& =2 \mathrm{l}_{1} \mathrm{l}_{2} \\
& \mathrm{k}_{1}=\mathrm{mR}-2 \mathrm{~m}^{2} \mathrm{q}-4 \mathrm{~m}^{2} \mathrm{t}, \\
& \mathrm{k}_{2}=\mathrm{R}^{2}+\mathrm{mR}+2 \mathrm{mRp}+2 \mathrm{~m}^{2} \mathrm{q} \\
& \mathrm{k} 3=4 \mathrm{~m} 2 \mathrm{c} 1 \mathrm{c} 2 \mathrm{c} 3 \mathrm{~s} 1 \mathrm{~s} 2 \mathrm{~s} 3, \mathrm{R}=\mathrm{r}^{2}+ \\
& \mathrm{l}_{1}^{2} \cos { }^{2} \theta+\mathrm{l}_{2}^{2} \mathrm{sin}^{2} \theta, \mathrm{p}=\mathrm{s} 12+ \\
& \mathrm{s} 22+\mathrm{s} 32 \\
& \mathrm{q}=\mathrm{s} 12 \mathrm{~s} 22+\mathrm{s} 12 \mathrm{~s} 32+ \\
& \mathrm{s} 22 \mathrm{~S} 32, \mathrm{t}=\mathrm{s} 12 \mathrm{~s} 22 \mathrm{~s} 32
\end{aligned}
$$

and $\mathrm{s}_{\mathrm{i}}, \mathrm{c}_{\mathrm{i}}$ stand for $\sinh \delta_{\mathrm{i}}, \cosh \delta_{\mathrm{i}}(\mathrm{i}=1,2,3)$, respectively. Electromagnetic vector potentials are given by: 


$$
\begin{aligned}
& A_{t 1}^{(1)}=\frac{m \cosh \delta_{1} \sinh \delta_{1}}{r^{2}+2 m \sinh ^{2} \delta_{1}+l_{1}^{2} \cos ^{2} \theta+l_{2}^{2} \sin ^{2} \theta}, \\
& A_{\phi 1}^{(1)}=m \sin ^{2} \theta \frac{l_{1} \sinh \delta_{1} \sinh \delta_{2} \cosh \delta_{3}-l_{2} \cosh \delta_{1} \cosh \delta_{2} \sinh \delta_{3}}{r^{2}+2 m \sinh ^{2} \delta_{1}+l_{1}^{2} \cos ^{2} \theta+l_{2}^{2} \sin ^{2} \theta}, \\
& A_{\psi 1}^{(1)}=m \cos ^{2} \theta \frac{l_{1} \cosh \delta_{1} \sinh \delta_{2} \sinh \delta_{3}-l_{2} \sinh \delta_{1} \cosh \delta_{2} \cosh \delta_{3}}{r^{2}+2 m \sinh ^{2} \delta_{1}+l_{1}^{2} \cos ^{2} \theta+l_{2}^{2} \sin ^{2} \theta}, \\
& A_{t 1}^{(2)}=\frac{m \cosh \delta_{2} \sinh \delta_{2}}{r^{2}+2 m \sinh ^{2} \delta_{2}+l_{1}^{2} \cos ^{2} \theta+l_{2}^{2} \sin ^{2} \theta}, \\
& A_{\phi 1}^{(2)}=m \sin ^{2} \theta \frac{l_{1} \cosh \delta_{1} \sinh \delta_{2} \cosh \delta_{3}-l_{2} \sinh \delta_{1} \cosh \delta_{2} \sinh \delta_{3}}{r^{2}+2 m \sinh ^{2} \delta_{2}+l_{1}^{2} \cos ^{2} \theta+l_{2}^{2} \sin ^{2} \theta}, \\
& A_{\psi 1}^{(2)}=m \cos ^{2} \theta \frac{l_{1} \sinh \delta_{1} \cosh \delta_{2} \sinh \delta_{3}-l_{2} \cosh _{1} \sinh \delta_{2} \cosh \delta_{3}}{r^{2}+2 m \sinh ^{2} \delta_{2}+l_{1}^{2} \cos ^{2} \theta+l_{2}^{2} \sin ^{2} \theta},
\end{aligned}
$$

$B_{t \phi}=-2 m \sin ^{2} \theta\left(l_{1} \sinh \delta_{1} \sinh \delta_{2} \cosh \delta_{3}-l_{2} \cosh \delta_{1} \cosh \delta_{2} \sinh \delta_{3}\right)\left(r^{2}+l_{1}^{2} \cos ^{2} \theta\right.$

$$
\left.+l_{2}^{2} \sin ^{2} \theta+m \sinh ^{2} \delta_{1}+m \sinh ^{2} \delta_{2}\right) /\left[\left(r^{2}+l_{1}^{2} \cos ^{2} \theta+l_{2}^{2} \sin ^{2} \theta\right.\right.
$$$$
\left.\left.+2 m \sinh ^{2} \delta_{1}\right)\left(r^{2}+l_{1}^{2} \cos ^{2} \theta+l_{2}^{2} \sin ^{2} \theta+2 m \sinh ^{2} \delta_{2}\right)\right] \text {, }
$$

$B_{t \psi}=-2 m \cos ^{2} \theta\left(l_{2} \sinh \delta_{1} \sinh \delta_{2} \cosh \delta_{3}-l_{1} \cosh \delta_{1} \cosh \delta_{2} \sinh \delta_{3}\right)\left(r^{2}+l_{1}^{2} \cos ^{2} \theta\right.$

$$
\begin{aligned}
& \left.+l_{2}^{2} \sin ^{2} \theta+m \sinh ^{2} \delta_{1}+m \sinh ^{2} \delta_{2}\right) /\left[\left(r^{2}+l_{1}^{2} \cos ^{2} \theta+l_{2}^{2} \sin ^{2} \theta\right.\right. \\
& \left.\left.+2 m \sinh ^{2} \delta_{1}\right)\left(r^{2}+l_{1}^{2} \cos ^{2} \theta+l_{2}^{2} \sin ^{2} \theta+2 m \sinh ^{2} \delta_{2}\right)\right],
\end{aligned}
$$

$B_{\phi \psi}=\frac{2 m \cosh \delta_{3} \sinh \delta_{3} \cos ^{2} \theta \sin ^{2} \theta\left(r^{2}+l_{1}^{2} \cos ^{2} \theta+l_{2}^{2} \sin ^{2} \theta+m \sinh ^{2} \delta_{1}+m \sinh ^{2} \delta_{2}\right)}{\left(r^{2}+l_{1}^{2} \cos ^{2} \theta+l_{2}^{2} \sin ^{2} \theta+2 m \sinh ^{2} \delta_{1}\right)\left(r^{2}+l_{1}^{2} \cos ^{2} \theta+l_{2}^{2} \sin ^{2} \theta+2 m \sinh ^{2} \delta_{2}\right)}$,

$1=\mathrm{R}^{3}+2 \mathrm{mpR} \mathrm{R}^{2}+4 \mathrm{~m}^{2} \mathrm{qR}+8 \mathrm{~m}^{3} \mathrm{t}$

$r_{ \pm}^{2}=m-\frac{1}{2} L_{1} \pm \frac{1}{2} \sqrt{L_{2}^{2}+4 m\left(m-L_{1}\right)}$

and we choose $G_{N}=\frac{1}{4} \pi$.

The physical quantities: ADM mass $\mathrm{M}$, three charges $\mathrm{Q}_{1}, \mathrm{Q}_{2}, \mathrm{Q}_{3}$ and two angular momenta $\mathrm{J}_{1}, \mathrm{~J}_{2}$, are given by

$$
\begin{aligned}
& \mathrm{M}=2 \mathrm{~m}\left(\cosh ^{2} \delta_{1}+\cosh ^{2} \delta_{2}+\cosh ^{2} \delta_{3}\right)-3 \mathrm{~m}, \\
& \mathrm{Q}_{1}=2 \mathrm{~m} \cosh \delta_{1} \sinh \delta_{1}, \\
& \mathrm{Q}_{2}=2 \mathrm{~m} \cosh \delta_{2} \sinh \delta_{2}, \\
& \mathrm{Q}_{3}=2 \mathrm{~m} \cosh \delta_{3} \sinh \delta_{3}, \\
& \mathrm{~J}_{1}=4 \mathrm{~m}\left(\mathrm{l}_{1} \cosh \delta_{1} \cosh \delta_{2} \cosh \delta_{3}-1_{2} \sinh \delta_{1} \sinh \delta_{2} \sinh \delta_{3}\right), \\
& \mathrm{J}_{2}=4 \mathrm{~m}\left(\mathrm{l}_{2} \cosh \delta_{1} \cosh \delta_{2} \cosh \delta_{3}-1_{1} \sinh \delta_{1} \sinh \delta_{2} \sinh \delta_{3}\right),
\end{aligned}
$$


$\mathrm{m}$ is the non-extremality parameter, $\delta_{1,2,3}$ are the boost parameters and $l_{1,2}$ are the angular momentum parameters.

\subsection{The first law of black-hole thermodynamics The}

entropy is given by [3]:

$$
\begin{gathered}
S=4 \pi m\left[\sqrt{2 m-\left(l_{1}-l_{2}\right)^{2}}\left(\cosh \delta_{1} \cosh \delta_{2} \cosh \delta_{3}+\sinh \delta_{1} \sinh \delta_{2} \sinh \delta_{3}\right)\right. \\
\left.+\sqrt{2 m-\left(l_{1}+l_{2}\right)^{2}}\left(\cosh \delta_{1} \cosh \delta_{2} \cosh \delta_{3}-\sinh \delta_{1} \sinh \delta_{2} \sinh \delta_{3}\right)\right] \\
\mathrm{d} S=\tilde{\Gamma}_{1} \mathrm{~d} Q_{1}+\tilde{\Gamma}_{2} \mathrm{~d} Q_{2}+\tilde{\Gamma}_{3} \mathrm{~d} Q_{3}+\tilde{\Gamma}_{4} \mathrm{~d} M+\tilde{\Gamma}_{5} \mathrm{~d} J_{1}+\tilde{\Gamma}_{6} \mathrm{~d} J_{2} .
\end{gathered}
$$

The first law is of the form

$$
\mathrm{dM}=\mathrm{T} \mathrm{dS}+8_{1} \mathrm{dQ}_{1}+8_{2} \mathrm{dQ}_{2}+8 \mathrm{dQ}_{3}+\bullet{ }_{1} \mathrm{dJ}_{1}+\cdot{ }_{2} \mathrm{dJ}_{2} .
$$

Once again, we need to invert a Jacobian matrix and find the derivatives of solution parameters with respect to the physical parameters, because we cannot invert the algebraic equations in (27) and find the solution parameters explicitly. The details are in appendix B. The result of this calculation is:

$$
\begin{aligned}
& T=\frac{\alpha \beta}{S}, \\
& \Phi_{i}=\frac{S_{, i}}{S} \quad(i=1,2,3), \\
& \Omega_{1}=-\pi \frac{\beta\left(l_{2}-l_{1}\right)-\alpha\left(l_{1}+l_{2}\right)}{S}, \\
& \Omega_{2}=-\pi \frac{\beta\left(l_{1}-l_{2}\right)-\alpha\left(l_{1}+l_{2}\right)}{S} .
\end{aligned}
$$

where

$$
\begin{aligned}
& \alpha=\sqrt{2 m-\left(l_{1}-l_{2}\right)^{2}}, \\
& \beta=\sqrt{2 m-\left(l_{1}+l_{2}\right)^{2}} .
\end{aligned}
$$

\subsection{Thermodynamic quantities derived from the metric}

Now, we make an independent check of the coefficients in the first law. Using the metric, we can calculate ${ }_{1}$,

$$
\begin{aligned}
\Omega_{1} & \left.\equiv \frac{-g_{t t}}{g_{\phi t}}\right|_{r=r_{+}, \theta=\frac{1}{2} \pi}, \\
& =\frac{R(R-2 m)}{-2 m \sin ^{2} \theta\left(l_{1} R c_{1} c_{2} c_{3}+l_{2}(2 m-R) s_{1} s_{2} s_{3}\right)} .
\end{aligned}
$$

$r=r_{+}, \quad=\frac{1}{2} \pi$

$R=-\frac{\left[\beta\left(l_{2}-l_{1}\right)-\alpha\left(l_{2}+l_{1}\right)\right] l_{2}}{\alpha-\beta}$, where $\mathrm{R}=$ $\mathrm{r}^{2}+1_{1}^{2} \cos ^{2}$ $\theta+1_{2}^{2} \sin ^{2}$ 
where

$\theta$. $\theta$

We consider the point on the outer horizon with,

so

$$
{ }_{1}=-2 \pi \frac{\beta\left(1_{2}-1_{1}\right)-\alpha\left(1_{2}+l_{1}\right)}{S} .
$$

This result is in agreement with the first law (30) except for a numerical factor. We can repeat the calculation for ${ }_{2}$. It is also in agreement with the first law. Now, let us make an independent check for $\kappa$, the surface gravity of the outer horizon [5]

$$
2 \pi T=\kappa=-\left.\frac{1}{2} \sqrt{-g^{r r} g^{t t}} \frac{\mathrm{d} \Sigma}{\mathrm{d} r}\right|_{r=r_{+}, \theta=\frac{1}{2} \pi},
$$

where

$$
\Sigma=g_{t t}-\frac{\left(g_{\phi t}+g_{\psi t}\right)^{2}}{g_{\phi \phi}+g_{\psi \psi}+2 g_{\phi \psi}} .
$$

To find $\kappa$, we need to calculate $\mathrm{g}^{\mathrm{rr}}, \mathrm{g}^{\mathrm{tt}}$ and put 6 into some manageable form. The details of these calculations are in appendix $\mathrm{B}$. The result is:

$$
\kappa=\frac{\sqrt{l_{2}^{2}+4 m\left(m-L_{1}\right)}}{\sqrt{\Delta+L_{1} k_{1}+L_{2} k_{2}+L_{3} k_{3}}} .
$$

At the horizon, $\mathrm{S}=4 \pi \sqrt{1}+\overline{\mathrm{L}_{1} \mathrm{k}_{1}+\mathrm{L}_{2} \mathrm{k}_{2}+\mathrm{L}_{3} \mathrm{k}_{3} \text {, so }}$

$$
\begin{array}{r}
\kappa=\frac{4 \pi \alpha \beta}{S} . \\
\Phi_{1}=A_{t 1}^{(1)}, \Phi_{2}=A_{t 1}^{(2)} \text { We can check the potentials } 8_{\mathrm{i}} \text { for the special case } \mathrm{l}_{1}=\mathrm{l}_{2}=0 . \text { In } \\
\text { this case, } \mathrm{r}^{2}=2 \mathrm{~m},+
\end{array}
$$

and.

\section{Conclusion}

Interpreting the surface area of a black hole as its entropy was one of the breakthroughs of black-hole thermodynamics. This made possible the analogy between the first law of blackhole mechanics $\left(\mathrm{dM}=\mathrm{T} \mathrm{dS}+\bullet \mathrm{dJ}+8_{\mathrm{i}} \mathrm{dQ}_{\mathrm{i}}\right)$ and the first law of thermodynamics.

In this paper I calculated the temperature, angular velocity and the potentials that enter the first law for two different black holes from toroidally compactified string theory. I did this using the expression for entropy as a starting point and taking derivatives using the chain rule to make a change of variables.

Then, as a check, I calculated the temperature and angular velocity directly using the metric. The values found using the two different methods are in agreement.

Note that in four dimensions, we used the formula ${ }^{\kappa}=-\frac{1}{2}\left(\mathrm{~d} g_{t t} / \mathrm{d} r\right)$, but in five 
dimensions we have to use the more general formula

\section{Appendix A}

$$
\kappa=-\left.\frac{1}{2} \sqrt{-g^{r r} g^{t t}} \frac{\mathrm{d}}{\mathrm{d} r}\left(g_{t t}-\frac{\left(g_{\phi t}+g_{\psi t}\right)^{2}}{g_{\phi \phi}+g_{\psi \psi}+2 g_{\phi \psi}}\right)\right|_{r=r_{+}, \theta=\frac{1}{2} \pi},
$$

Note that we can write $\mathrm{S}$ in the form

$K=\cosh \delta_{1} \cosh \delta_{2} \cosh \delta_{3} \cosh \delta_{4}+\sinh \delta_{1} \sinh \delta_{2} \sinh \delta_{3} \sinh \delta_{4}$

$L=\cosh \delta_{1} \cosh \delta_{2} \cosh \delta_{3} \cosh \delta_{4}-\sinh \delta_{1} \sinh \delta_{2} \sinh \delta_{3} \sinh \delta_{4}$.

$$
S=16 \pi\left(m^{2} K+m \sqrt{m^{2}-l^{2}} L\right)
$$

To determine the coefficients $0_{\mathrm{i}}$, we have to invert the following matrix:

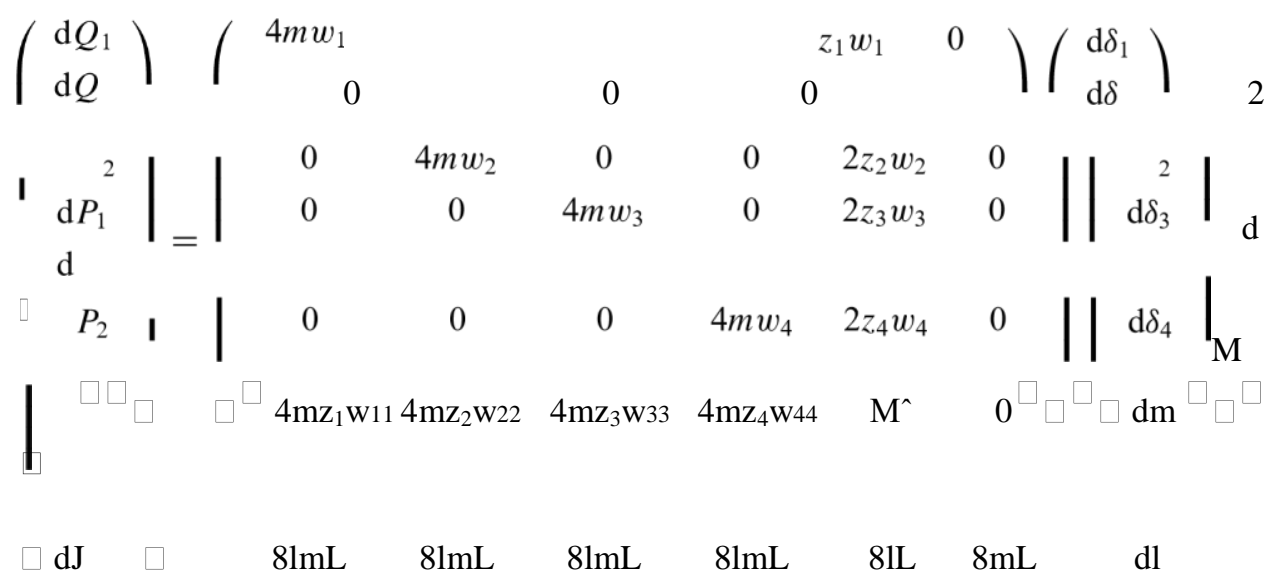

where

$$
\begin{aligned}
w_{i} & =\cosh 2 \delta_{i}, \\
z_{i} & =\tanh 2 \delta_{i}, \\
\hat{M} & =\frac{M}{m}, \\
L_{i} & =\frac{\partial L}{\partial \delta_{i}} \quad(i=1, \ldots, 4) .
\end{aligned}
$$

(A.4) The result is 
where

$$
\begin{aligned}
& \left(\begin{array}{l}
\mathrm{d} \delta_{1} \\
\mathrm{~d} \delta_{2} \\
\mathrm{~d} \delta_{3} \\
\mathrm{~d} \delta_{4} \\
\mathrm{~d} m \\
\mathrm{~d} l
\end{array}\right)=\frac{1}{B}\left(\begin{array}{cccccc}
\frac{B}{4 m w_{1}}+z_{1}^{2} & z_{1} z_{2} & z_{1} z_{3} & z_{1} z_{4} & -z_{1} & 0 \\
z_{2} z_{1} & \frac{B}{4 m w_{2}}+z_{2}^{2} & z_{2} z_{3} & z_{2} z_{4} & -z_{2} & 0 \\
z_{3} z_{1} & z_{3} z_{2} & \frac{B}{4 m w_{3}}+z_{3}^{2} & z_{3} z_{4} & -z_{3} & 0 \\
z_{4} z_{1} & z_{4} z_{2} & z_{4} z_{3} & \frac{B}{4 m w_{4}}+z_{4}^{2} & -z_{4} & 0 \\
-2 m z_{1} & -2 m z_{2} & -2 m z_{3} & -2 m z_{4} & 2 m & 0 \\
\frac{u_{1}}{L} & \frac{u_{2}}{L} & \frac{u_{3}}{L} & \frac{u_{4}}{L} & \frac{l P_{L}}{L}-2 l & \frac{B}{8 m L}
\end{array}\right) \\
& \times\left(\begin{array}{c}
\mathrm{d} Q_{1} \\
\mathrm{~d} Q_{2} \\
\mathrm{~d} P_{1} \\
\mathrm{~d} P_{2} \\
\mathrm{~d} M \\
\mathrm{~d} J
\end{array}\right)
\end{aligned}
$$


where

(A.6) Now,

$$
u_{i}=-z_{i} l P_{L}+2 z_{i} l L-\frac{l L_{i} B}{4 m w_{i}} \quad(i=1, \ldots, 4 \quad),
$$

$$
\begin{aligned}
& P_{L}=L_{1} z_{1}+L_{2} z_{2}+L_{3} z_{3}+L_{4} z_{4}, \\
& B=2 M-4 m\left(w_{1} z_{1}^{2}+w_{2} z_{2}^{2}+w_{3} z_{3}^{2}+w_{4} z_{4}^{2}\right) .
\end{aligned}
$$

using this matrix, we can

calculate the $0_{\mathrm{i}}$ 's defined in (9)

$$
\begin{aligned}
\Gamma_{5} & =\frac{S}{4 \sqrt{m^{2}-l^{2}}}, \\
\Gamma_{6} & =\frac{-2 \pi l}{\sqrt{m^{2}-l^{2}}}, \\
\Gamma_{i} & =\frac{-S_{i}}{4 \sqrt{m^{2}-l^{2}}} \quad(i=1, \ldots, 4 \quad),
\end{aligned}
$$

where

$$
\begin{aligned}
& \mathrm{T}=1 / 0_{5}, \\
& \square=-0_{6} / 0_{5}, \\
& 8_{\mathrm{i}}=-0_{\mathrm{i}} / 0_{5} \quad(\mathrm{i}=1, \ldots, 4)
\end{aligned}
$$

and $\mathrm{S}_{\mathrm{i}} \equiv \partial \mathrm{S} / \partial \delta_{\mathrm{i}}$.

\section{Appendix B}

Appendix B.1. Finding the $0^{\sim}$ 's

To find the derivatives of boosts with respect to physical variables $\left(\partial \delta_{1} / \partial \mathrm{Q}_{2}\right.$ etc) we need to invert the following matrix:

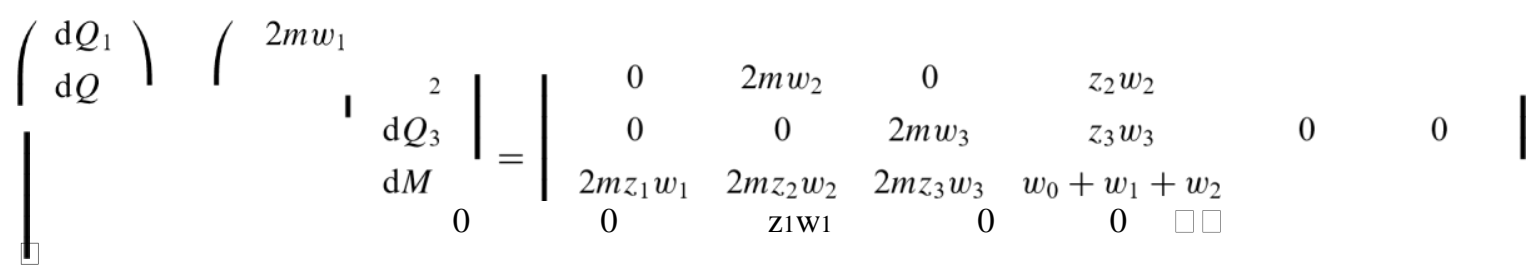


where

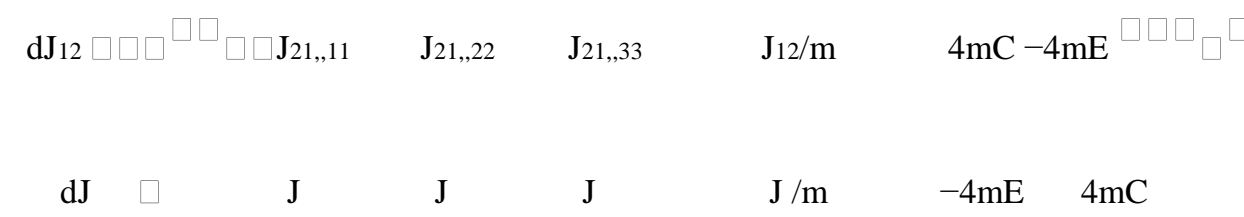

$\mathrm{d} \delta 2 \mathrm{~d} \delta$

$\square \square \square \square \mathrm{d} \delta_{1}{ }^{3} \square \square \square \square \square \square$

$\times \square \square \square \mathrm{dm}$

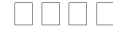

(B.1)

$\mathrm{dl}_{1} \mathrm{dl}_{2} \mathrm{~W}_{\mathrm{i}}$

$\cosh 2 \delta_{\mathrm{i}}$

$(\mathrm{i}=$

$1,2,3)$

$\mathrm{Z}_{\mathrm{i}}=\tanh 2 \delta_{\mathrm{i}} \quad(\mathrm{i}=1,2,3)$,

(B.2) $\mathrm{C}=\cosh \delta_{1} \cosh \delta_{2} \cosh \delta_{3}$,

$\mathrm{E}=\sinh \delta_{1} \sinh \delta_{2} \sinh \delta_{3}$.

The result is 


$$
\begin{aligned}
& 1462 \text { E Sermutlu } \\
& \left(\begin{array}{l}
\mathrm{d} \delta_{1} \\
\mathrm{~d} \delta_{2}
\end{array}\right) \quad\left(\begin{array}{llll}
z_{2} z_{1} & \underset{w_{2}}{U}+z_{2}^{2} & z_{2} z_{3} & -z_{2}
\end{array}\right.
\end{aligned}
$$

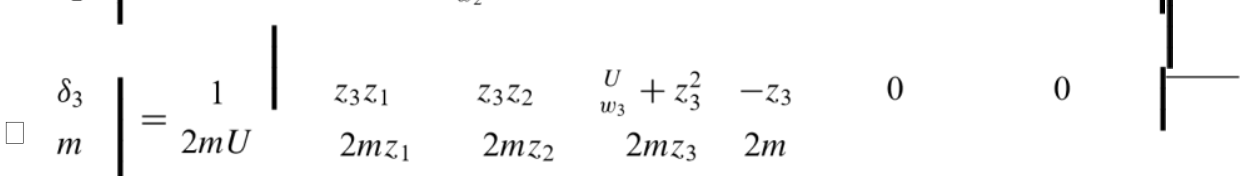

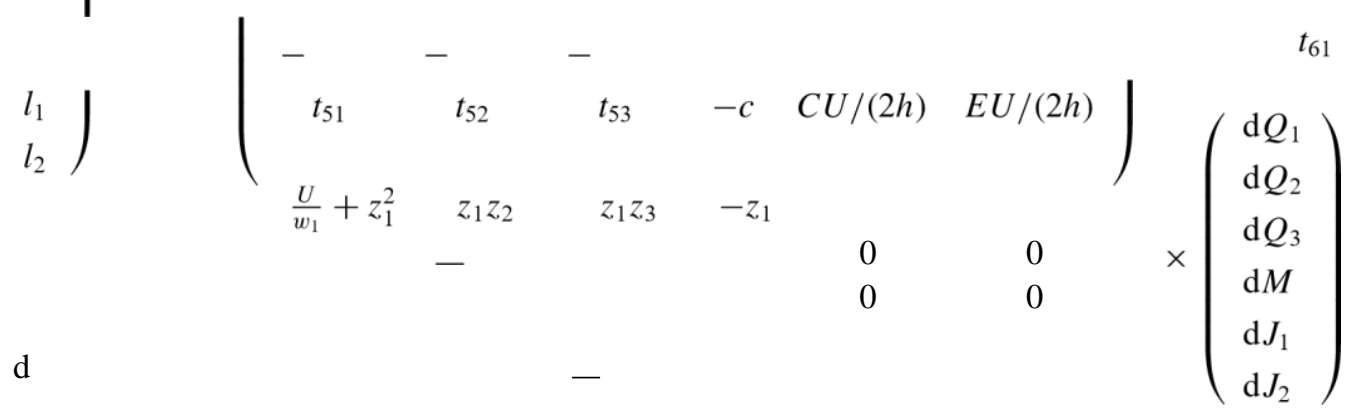

$$
\begin{aligned}
& \square \mathrm{d} \\
& \begin{array}{clll}
- & 0 & 0 & \square
\end{array}
\end{aligned}
$$

$\mathrm{t}_{63} \quad \mathrm{~d} \quad \mathrm{EU} /(2 \mathrm{~h}) \quad \mathrm{CU} /(2 \mathrm{~h})$

(B.3)

where 
where

$$
\begin{aligned}
& U=\left(w_{1}+w_{2}+w_{3}-w_{1} z_{1}^{2}-w_{2} z_{2}^{2}-w_{3} z_{3}^{2} \quad\right), \\
& P_{E}=E_{1} z_{1}+E_{2} z_{2}+E_{3} z_{3}, \\
& P_{C}=C_{1} z_{1}+C_{2} z_{2}+C_{3} z_{3}, \\
& h=(C-E)(C+E), \\
& a=\frac{E l_{1}+C l_{2}}{h}, \\
& \text { Using these } b=\frac{C l_{1}+E l_{2}}{h} \text {, }
\end{aligned}
$$

as follows:

$$
\begin{array}{rlrl}
c & =2 l_{1}+a P_{E}-b P_{C}, & & \\
d & =2 l_{2}+b P_{E}-a P_{C}, & \tilde{\Gamma}_{i}=\frac{-S_{i}}{\alpha \beta}, \\
t_{5 i}=\frac{U}{w_{i}}\left(E_{i} a-C_{i} b\right)+z_{i} c, & \tilde{\Gamma}_{4}=\frac{S}{\alpha \beta}, \\
t_{6 i}=\frac{U}{w_{i}}\left(E_{i} b-C_{i} a\right)+z_{i} d . & \tilde{\Gamma}_{5}=\pi\left(\frac{l_{2}-l_{1}}{\alpha}-\frac{l_{1}+l_{2}}{\beta}\right),
\end{array}
$$

(B.6)

$$
\tilde{\Gamma}_{6}=\pi\left(\frac{l_{1}-l_{2}}{\alpha}-\frac{l_{1}+l_{2}}{\beta}\right) \text {. }
$$

\section{Appendix B.2. Finding $\mathrm{\kappa}$}

We know that $\mathrm{g}^{\mathrm{rt}}=\mathrm{g}_{\mathrm{rr}}{ }^{-1}$ and $\mathrm{g}^{\mathrm{tt}}=\left(\mathrm{g}_{\varphi \varphi} \mathrm{g}_{\psi \psi}-\mathrm{g}_{\varphi \psi}{ }^{2}\right) / \mathrm{D}$, where

$$
\begin{aligned}
& D=\frac{\operatorname{Det}\left(g_{i j}\right)}{g_{r r} g_{\theta \theta}} \\
& \mathrm{D}=\mathrm{g}_{\mathrm{tt}}\left(\mathrm{g}_{\varphi \varphi} \mathrm{g}_{\psi \psi}-\mathrm{g}_{\varphi \mathrm{t} 2}\right)+2 \mathrm{~g}_{\varphi \psi} \mathrm{g}_{\varphi \mathrm{t}} \mathrm{g}_{\psi \mathrm{t}}-\mathrm{g}_{\psi \mathrm{t} 2} \mathrm{~g}_{\varphi \varphi}-\mathrm{g}_{\varphi \mathrm{t} 2} \mathrm{~g}_{\psi \psi} .
\end{aligned}
$$

After some algebra, we find that

$$
\begin{aligned}
& \mathrm{D}=\cos ^{2} \theta \sin ^{2} \theta\left[(2 \mathrm{~m}-\mathrm{R}) \mathrm{R}+\mathrm{L}_{2}\left(\cos ^{2} \theta-\sin ^{2} \theta\right)(\mathrm{R}-\mathrm{m})-\mathrm{L}_{1} \mathrm{~m}+\mathrm{L}_{2}{ }_{2} \cos ^{2} \theta \sin ^{2} \theta\right] \\
& =-\cos 2 \theta \sin 2 \theta 11 / 3 \mathrm{r}^{2} \mathrm{~g}_{\mathrm{rr}-1} \\
& g_{\phi \phi} g_{\psi \psi}-g_{\phi \psi}^{2}=\cos ^{2} \theta \sin ^{2} \theta \Delta^{-1 / 3}\left[\Delta+k_{1} L_{1}+\left(\sin ^{2} \theta-\cos ^{2} \theta\right) k_{2} L_{2}\right. \\
& \left.\quad+k_{3} L_{3}-\cos ^{2} \theta \sin ^{2} \theta L_{2}^{2}(2 m+2 m p+R)\right] . \\
& -g^{t t} g^{r r}=\frac{\Delta+k_{1} L_{1}+\left(\sin ^{2} \theta-\cos ^{2} \theta\right) k_{2} L_{2}+k_{3} L_{3}-\cos ^{2} \theta \sin ^{2} \theta L_{2}^{2}(2 m+2 m p+R)}{r^{2} \Delta^{2 / 3}}
\end{aligned}
$$


1464

\section{E Sermutlu}

$$
\begin{gathered}
\begin{aligned}
\Sigma=\Delta^{1 / 3}\left[R(2 m-R)+R\left(\cos ^{2} \theta-\sin ^{2} \theta\right) L_{2}-2 m\left(\cos ^{2} \theta l_{1}+\sin ^{2} \theta l_{2}\right)^{2}\right] /[\Delta \\
+\left(L_{1} k_{1}+L_{3} k_{3}\right)\left(\sin ^{4} \theta+\cos ^{4} \theta\right)+L_{2} k_{2}\left(\sin ^{2} \theta-\cos ^{2} \theta\right) \\
\left.+2 \sin ^{2} \theta \cos ^{2} \theta\left(L_{1} k_{3}+L_{3} k_{1}\right)\right]
\end{aligned} \\
\text { (B.10) } \Sigma=\Delta^{1 / 3}\left[R(2 m-R)+R\left(\cos ^{2} \theta-\sin ^{2} \theta\right) L_{2}-2 m\left(\cos ^{2} \theta l_{1}+\sin ^{2} \theta l_{2}\right)^{2}\right] /[\Delta
\end{gathered}
$$

$$
\begin{aligned}
& +\left(L_{1} k_{1}+L_{3} k_{3}\right)\left(\sin ^{4} \theta+\cos ^{4} \theta\right)+L_{2} k_{2}\left(\sin ^{2} \theta-\cos ^{2} \theta\right) \\
& \left.+2 \sin ^{2} \theta \cos ^{2} \theta\left(L_{1} k_{3}+L_{3} k_{1}\right)\right] .
\end{aligned}
$$

Note that at the

horizon, $R=m-\frac{1}{2} L_{2}+\frac{1}{2} \sqrt{ } L_{2}^{2}+4 m\left(m-L_{1}\right)$ and $\Sigma={ }_{0}$. We find

$$
\kappa=\frac{\sqrt{l_{2}^{2}+4 m\left(m-L_{1}\right)}}{\sqrt{\Delta+L_{1} k_{1}+L_{2} k_{2}+L_{3} k_{3}}} .
$$




\section{Acknowledgments}

I would like to thank Mirjam Cvetic for giving me the idea, expanding it through stimulating discussions, for her guidance, helpful comments and her interest throughout the work. I would also like to thank Finn Larsen and Metin Gurses for helpful suggestions. This work was supported by the BDP program of TUBITAK (Scientific and Technical Research Council of Turkey).

\section{References}

[1] Bardeen J M, Carter B and Hawking S W 1973 Commun. Math. Phys. 31161

[2] Smarr L 1973 Phys. Rev. Lett. 3071

[3] Cvetic M and Youm D 1996 Phys. Rev. D 54 2612-20

[4] Cvetic M and Youm D 1996 Nucl. Phys. B 476 118-32

[5] Wald R M 1984 General Relativity (Chicago, IL: University of Chicago Press) section 12.5 